\title{
Management of antipsychotic treatment discontinuation and interruptions using model-based simulations
}

This article was published in the following Dove Press journal:

Clinical Pharmacology:Advances and Applications

13 July 2012

Number of times this article has been viewed

\author{
Mahesh N Samtani' \\ John J Sheehan ${ }^{2}$ \\ Dong-Jing $\mathrm{Fu}^{2}$ \\ Bart Remmerie ${ }^{3}$ \\ Jennifer Kern Sliwa ${ }^{2}$ \\ Larry Alphs ${ }^{2}$
}

'Janssen Research and Development, Raritan, NJ, USA; 'ªnssen Scientific Affairs, Titusville, NJ, USA; ${ }^{3}$ Janssen

Research and Development, Division of Janssen Pharmaceutica, Beerse, Belgium
Correspondence: Mahesh N Samtani Johnson and Johnson Pharmaceutical $R$ and D, Clinical Pharmacology, Advanced Modeling and Simulation 920 Route 202, Raritan, NJ 08869, USA

$\mathrm{Tel}+\mathrm{I} 9087045367$

Fax + I 9089272573

Email msamtani@its.jnj.com
Background: Medication nonadherence is a well described and prevalent clinical occurrence in schizophrenia. These pharmacokinetic model-based simulations analyze predicted antipsychotic plasma concentrations in nonadherence and treatment interruption scenarios and with treatment reinitiation.

Methods: Starting from steady state, pharmacokinetic model-based simulations of active moiety plasma concentrations of oral, immediate-release risperidone $3 \mathrm{mg}$ /day, risperidone long-acting injection $37.5 \mathrm{mg} / 14$ days, oral paliperidone extended-release $6 \mathrm{mg} /$ day, and paliperidone palmitate $117 \mathrm{mg}$ ( $75 \mathrm{mg}$ equivalents)/28 days were assessed under three treatment discontinuation/ interruption scenarios, ie, complete discontinuation, one week of interruption, and four weeks of interruption. In the treatment interruption scenarios, pharmacokinetic simulations were performed using medication-specific reinitiation strategies.

Results: Following complete treatment discontinuation, plasma concentrations persisted longest with paliperidone palmitate, followed by risperidone long-acting injection, while oral formulations exhibited the most rapid decrease. One week of oral paliperidone or risperidone interruption resulted in near complete elimination from the systemic circulation within that timeframe, reflecting the rapid elimination rate of the active moiety. After 1 and 4 weeks of interruption, minimum plasma concentrations were higher with paliperidone palmitate than risperidone long-acting injection over the simulated period. Four weeks of treatment interruption followed by reinitiation resulted in plasma levels returning to predicted therapeutic levels within 1 week.

Conclusion: Due to the long half-life of paliperidone palmitate (25-49 days), putative therapeutic plasma concentrations persisted longest in simulated cases of complete discontinuation or treatment interruption. These simulations may help clinicians better conceptualize the impact of antipsychotic nonadherence on plasma concentrations, and the impact of medication-specific reinitiation strategies after intermittent nonadherence.

Keywords: paliperidone palmitate, risperidone, long-acting injection, pharmacokinetics, nonadherence, plasma concentrations

\section{Introduction}

The standard of care for patients with schizophrenia includes prompt initiation of antipsychotic treatment. ${ }^{1-3}$ However, a major barrier to the effective treatment of patients with schizophrenia is nonadherence to the medication regimen, with estimated one-year rates of treatment discontinuation or interruption ranging from $40 \%$ to $75 \%$. $^{4-6}$ Individual patients discontinue or interrupt treatment due to a variety of factors, including a lack of insight into their illness, forgetfulness, lack of social support, tolerability issues, conscious choice, and refractory or nonresponsive symptomatology. ${ }^{7}$ 
Complete discontinuation or interruption of treatment can have severe ramifications, including relapse and the need for hospitalization. ${ }^{8-10}$ Even short periods (ie, $\leq 2$ weeks) of nonadherence with oral antipsychotic therapy increased the risk of a psychotic exacerbation, with hazard ratios ranging from 3.7 to 28.5 , depending on the severity of medication nonadherence, ${ }^{11}$ and the risk of hospitalization increased nearly twofold. ${ }^{12}$

Although patients can achieve improvement in psychotic symptoms with daily oral medication, poor adherence is a significant hurdle to achieving favorable outcomes. ${ }^{13}$ In patients with a history of nonadherence, the American Psychiatric Association practice guidelines recommend the use of long-acting injectable antipsychotics. ${ }^{1}$ In addition, the guidelines state that for patients who are prone to forget doses or who are intermittently nonadherent with treatment, drugs with slower rates of metabolism may be used preferentially. ${ }^{1}$ After professional administration of a long-acting injectable formulation, patients do not need to remember to take their daily oral antipsychotic medication during the dosing period. Importantly, in the event of symptom recurrence, the clinician and caregiver will know the patient's adherence status and can correctly attribute the symptoms to medication failure or nonadherence. Long-acting injectable agents provide more stable drug exposure than daily formulations. Moreover, the long apparent half-lives for these products potentially allow more time to follow-up if a dose is delayed or missed, reducing the consequences of treatment interruption, such as risk of relapse and rehospitalization. ${ }^{7,8,14-16}$ Data from recurrence prevention trials suggest that the median time to relapse with long-acting agents is longer than with oral extended-release formulations, and median times to relapse of 62 days have been reported following a switch from oral paliperidone extended-release to placebo $^{17}$ and of 163 days following a switch from paliperidone palmitate long-acting injectable suspension to placebo. ${ }^{18,19}$ This increased time to relapse among patients switched from paliperidone palmitate to placebo may have resulted from persistent drug exposure, and a residual therapeutic effect, for weeks after the switch from a long-acting injectable to placebo.

Limited experimental data usually restrict the ability to visualize pharmacokinetic profiles following treatment discontinuation, or interruption and treatment reinitiation that may occur clinically in a patient receiving orally administered or long-acting injectable antipsychotic agents. However, pharmacokinetic models have been used to simulate a wide variety of situations that could be encountered clinically to inform prescriber judgment. ${ }^{20}$ To help address these situations encountered in every day practice, we simulated three scenarios using paliperidone and risperidone. We selected these scenarios and four formulations of paliperidone and risperidone because of: the availability of pharmacokinetic models that permit simulation of treatment discontinuation/interruption scenarios; the pharmacodynamic relationship between the atypical antipsychotics, risperidone and paliperidone (ie, paliperidone, also known as 9-hydroxy-risperidone, is the metabolite of risperidone, with both risperidone and 9-hydroxyrisperidone being active moieties), and the availability of both agents as oral and long-acting injectable formulations. Selecting two long-acting injectable formulations with substantially different release characteristics permits comparison with oral drugs and between formulations. These pharmacokinetic model-based discontinuation, interruption, and reinitiation simulations provide clinicians with a visual depiction of the plasma concentration profiles that may be anticipated with the four formulations of these two agents that have substantially different half-lives and formulation release properties.

Additional objectives of this analysis were to understand the comparative pharmacokinetics of the four paliperidone and risperidone formulations. Therefore, we also provide a brief review of the pharmacokinetic properties of these four formulations. The two oral formulations that were assessed differ in their release profiles in that risperidone displays an immediate-release profile (usually within one hour) whereas paliperidone is an extended-release tablet that uses the OROS delivery technology and displays an ascending release profile that lasts approximately 24 hours. $^{21,22}$ The extended-release profile of oral paliperidone enables initiation of treatment with an effective dose without the need for initial dose titration. ${ }^{23}$ With multiple dosing, the oral risperidone immediate-release formulation exhibits daily peak-trough fluctuations whereas the extended-release formulation of oral paliperidone exhibits less fluctuation in steady-state plasma concentrations. ${ }^{21,22,24,25}$

The long-acting injectable formulations of risperidone and paliperidone differ considerably from their respective oral formulations as well as between each other. The longacting injectable formulation of risperidone (Risperdal ${ }^{\circledR}$ Consta $^{\mathrm{TM}}$; Janssen Pharmaceuticals, Inc; Titusville, NJ) uses biodegradable microspheres that are loaded with risperidone and suspended in aqueous diluent. ${ }^{26,27}$ This formulation is characterized by no substantial release of risperidone immediately following the injection. After a single intramuscular injection of risperidone long-acting injection, there is a small initial release of the drug ( $<1 \%$ of the dose) followed by a lag time of 3 weeks. ${ }^{26,27}$ The main release of the drug starts from 3 weeks onwards, is maintained for 4-6 weeks, and subsides 
by 7 weeks following intramuscular injection. ${ }^{26,27}$ Therefore, oral antipsychotic supplementation must be given during the first 3 weeks of treatment with risperidone long-acting injection to maintain therapeutic plasma concentrations until the main release of risperidone from the injection site has begun. ${ }^{26,27}$ Steady-state plasma concentrations are reached after four injections of risperidone long-acting injection and are maintained for 4-6 weeks after the last injection.

Paliperidone palmitate long-acting injection (Invega ${ }^{\circledR}$ Sustenna $^{\text {TM }}$ Janssen Pharmaceuticals, Inc ) is formulated as an aqueous suspension with a precisely milled particle size distribution that has sustained-release properties and permits once monthly (every 4 weeks) administration. Based on its extremely low solubility in water, paliperidone palmitate dissolves slowly at the injection site after intramuscular injection, is then hydrolyzed to paliperidone, and displays relatively constant plasma concentrations of paliperidone. ${ }^{28}$ Following administration of the first injection of paliperidone palmitate long-acting injectable suspension, drug release begins as early as day 1 and lasts for as long as 126 days. ${ }^{29}$ The median half-life is in the range of 25-49 days over the dose range of 39-234 mg (doses may also be expressed in mg equivalents [mg eq] of pharmacologically active paliperidone, with $39,78,117,156$, and $234 \mathrm{mg}$ of paliperidone palmitate corresponding to $25,50,75,100$, and $150 \mathrm{mg}$ eq of paliperidone).$^{30} \mathrm{~A}$ paliperidone palmitate initiation regimen with two intramuscular deltoid injections of $234 \mathrm{mg}$ on day 1 and $156 \mathrm{mg}$ on day 8 is recommended to attain therapeutic concentrations quickly. ${ }^{29,31}$ No oral supplementation is required to initiate paliperidone palmitate treatment.

\section{Materials and methods Risperidone, paliperidone, and paliperidone palmitate dose selection}

Pharmacokinetic model-based simulations were performed using oral risperidone, risperidone long-acting injection, oral paliperidone extended-release, and paliperidone palmitate long-acting injection. The dose of oral risperidone ( $3 \mathrm{mg} /$ day) used for the simulations results in active moiety (risperidone plus paliperidone) exposure similar to that of risperidone long-acting injection $37.5 \mathrm{mg} / 2$ weeks. ${ }^{32}$ Risperidone longacting injection $37.5 \mathrm{mg} / 2$ weeks potentially attains an active moiety steady-state exposure similar to that of paliperidone palmitate $117 \mathrm{mg} / 4$ weeks. ${ }^{33}$ The $117 \mathrm{mg}$ dose of injectable paliperidone palmitate and the $6 \mathrm{mg}$ oral paliperidone extended-release dose represent the recommended maintenance doses listed in the product package inserts and provide similar exposures for paliperidone..$^{29,33}$
It is important to note that the doses used in these pharmacokinetic model-based simulations do not represent dose equivalency across formulations because multistep comparisons of the above approximations can result in compounded deviation, and specific pharmacokinetic parameters (eg, peak-to-trough fluctuation) vary by formulation.

\section{Scenarios addressed in pharmacokinetic model-based simulations}

Pharmacokinetic simulations were performed under three scenarios (Table 1). The three simulated treatment discontinuation and interruption scenarios assumed that subjects were at steady state and received their last dose of orally administered risperidone or paliperidone on the previous day (day -1), their last injection of risperidone long-acting injection 2 weeks prior, and their last injection of paliperidone palmitate 4 weeks prior to the day of discontinuation/interruption, thus assuming discontinuation at the subsequent next administration time point of each individual formulation. In the treatment interruption scenarios, pharmacokinetic simulations were performed using medication-specific reinitiation strategies (Table 1).

\section{Models for pharmacokinetic simulations}

The pharmacokinetics of the active moiety after oral risperidone administration has been described using a twocompartment disposition model with linear elimination from the central compartment. ${ }^{25}$ Oral absorption was modeled

Table I Scenarios analyzed in pharmacokinetic model-based simulations

\begin{tabular}{ll}
\hline Scenario I & $\begin{array}{l}\text { Complete treatment discontinuation without reinitiation } \\
\text { of medication }\end{array}$ \\
Scenario 2 & One week of treatment interruption, followed by \\
& treatment reinitiation at the following doses: \\
& - Oral risperidone $3 \mathrm{mg}$ once daily \\
& - Risperidone LAI $37.5 \mathrm{mg}$ every 2 weeks \\
& - Oral paliperidone ER $6 \mathrm{mg}$ once daily \\
& - Paliperidone palmitate $1 \mathrm{I} 7 \mathrm{mg}$ (75 $\mathrm{mg}$ eq) \\
& every 4 weeks \\
Scenario 3 & Four weeks of treatment interruption, followed by \\
& treatment reinitiation at the following doses: \\
& - Oral risperidone $3 \mathrm{mg}$ once daily \\
& - Risperidone LAl $37.5 \mathrm{mg}$ every 2 weeks AND \\
& oral risperidone $3 \mathrm{mg}$ once daily supplementation \\
& administered from days 28 to 49 (ie, weeks $4-7$ from \\
& time of interruption) \\
- Oral paliperidone ER $6 \mathrm{mg}$ once daily & Paliperidone palmitate II $\mathrm{mg}$ (75 mg eq) \\
& administered on days 28 and 35 (ie, weeks 4 and \\
& 5 from time of interruption) and every 4 weeks \\
& thereafter
\end{tabular}

Abbreviations: eq, equivalent; ER, extended-release; LAI, long-acting injection. 
using a first-order process with a lag time from the gut to the central compartment.

The pharmacokinetics of the extended-release oral formulation of paliperidone have been described using a two-compartment disposition model with linear elimination from the central compartment. ${ }^{24}$ Oral absorption was modeled with a sequential zero order input into a depot compartment and first-order absorption, with a lag time from the depot to the central compartment. The relatively faster absorption of paliperidone from the oral route allowed identification of the distributive peripheral compartment, which is not discernible in the flip-flopped paliperidone palmitate pharmacokinetic model (see below).

A compartmental model has also been developed for intramuscular risperidone long-acting injection which includes a one-compartment disposition submodel characterized by clearance and volume of distribution. ${ }^{34}$ The plasma profile of the active moiety after intramuscular administration of risperidone long-acting injection is extremely complex because of the immediate release of a small amount of nonencapsulated risperidone followed by two sustained-release processes differing in their rate of release along with variable delay in initiation of release. Therefore, the model has three parallel absorption pathways with five absorption compartments which describe the immediate pathway and a fast and a slow sustained-release pathway.

Finally, a one-compartment model with first-order elimination has been used to describe the pharmacokinetics of paliperidone after intramuscular administration of its palmitate ester. ${ }^{28}$ The absorption component of the model allows a fraction of the dose to enter the central compartment relatively quickly via a zero-order process. After a certain lag time, the remaining fraction of the dose enters the systemic circulation via a first-order process that determines the shape of the plasma concentration-time curve following intramuscular injection.

For all the models described above, the final parameters from the published models, ${ }^{24,25,28,34}$ including all significant subject covariates, were used for simulating the pharmacokinetic exposure for a typical subject. Drug-drug interactions were not considered as part of this simulation exercise. The implementation of these simulations in Nonmem ${ }^{\circledR}$ software, along with the respective parameter estimates, is illustrated through control streams (codes) in Appendix 1.

\section{Model-based simulations}

Previously published pharmacokinetic models for paliperidone palmitate, oral paliperidone extended-release, risperidone long-acting injection, and oral risperidone were utilized as the simulation machinery for this analysis. ${ }^{24,25,28,34}$ Nonmem version VI (GloboMax, Icon Development Solutions, Ellicott City, MD) with an Intel Fortran 10 compiler was used to conduct all pharmacokinetic simulations. Visualization of results was performed using S-Plus ${ }^{\circledR} 6.0$ professional release 2 software (Insightful Corporation, Seattle, WA).

Pharmacokinetic simulations were used to predict the plasma concentrations over an 8-9-week period following treatment discontinuation (scenario 1) or interruption (scenarios 2 and 3). The simulations performed in this exercise are deterministic in nature (ie, between-subject variability and residual variability were not included in the simulations because the objective of this analysis was to visualize the typical pharmacokinetic profiles under various noncompliance situations). In the case of complete treatment discontinuation for each formulation (scenario 1), the number of days until the active moiety plasma concentrations fell below $2 \mathrm{ng} / \mathrm{mL}$ was calculated. Simulated profiles for the four formulations were also used to determine the lowest concentrations due to noncompliance $\left(\mathrm{C}_{\text {min,discontinuation }}\right)$ as a percentage of the steady-state minimum concentrations $\left(\mathrm{C}_{\text {min,steady state }}\right)$ for each treatment in scenario 2 (one week of treatment interruption with reinitiation at day 7) and scenario 3 (4 weeks of treatment interruption with reinitiation at day 28 ).

\section{Results \\ Scenario I: complete treatment discontinuation}

Following discontinuation of oral risperidone or oral paliperidone extended-release on day-1, the active-moiety concentrations decreased substantially within a few days (Figure 1). Two weeks following the last injection of risperidone long-acting injection, the concentration of the active moiety continued to approximate the steady-state concentration for about 3-5 weeks due to the delay in release (3-week lag time between last injection and last release phase). After this lag phase, there was a subsequent and rapid decline of the concentration of the active moiety, with near zero concentrations reached by week 5 (Figure 1). The slowest decline of active moiety concentration was observed with paliperidone palmitate (Figure 1).

The mean number of days needed for the active moiety plasma concentrations of each formulation to approach $2 \mathrm{ng} / \mathrm{mL}$ is depicted in Figure 2. Complete discontinuation of paliperidone palmitate resulted in a steady and slow decline of the concentration of the active moiety over the simulation period 


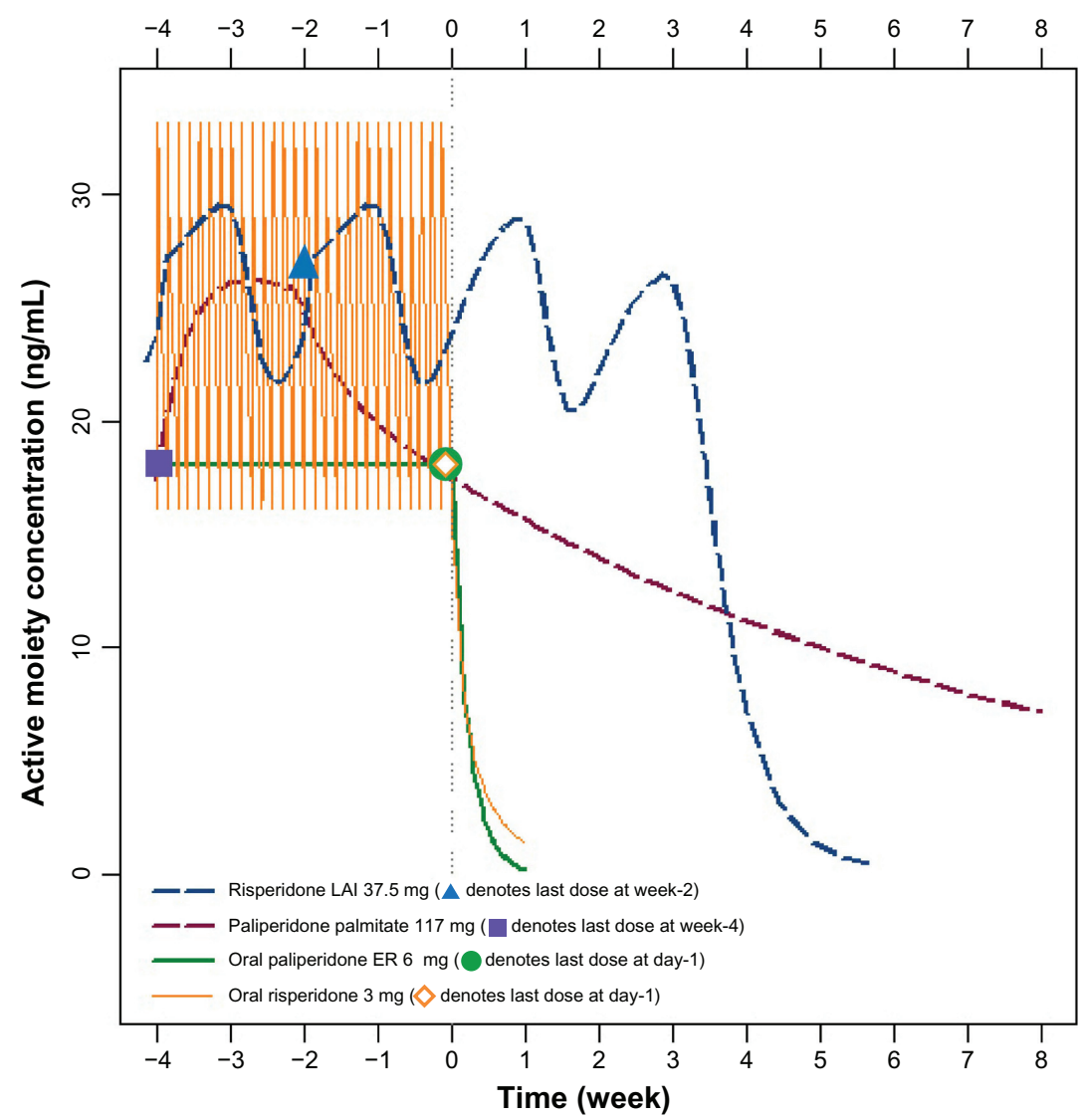

Figure I Active moiety concentrations following complete treatment discontinuation (week 0 denotes administration time of next scheduled dose, which was missed). Notes: Within a few days of oral risperidone or oral paliperidone ER discontinuation, there was a substantial decrease in the concentration of the active moiety. Two weeks following the last injection of risperidone LAl, the concentration of the active moiety continued to approximate the steady-state concentration for approximately $3-4$ weeks due to the delay in release (3-week lag time between last injection and last release phase). After this lag phase, there was a subsequent and rapid decline of the concentration of the active moiety, with near zero concentrations reached by week 5 . As compared with the other formulations, discontinuation of paliperidone palmitate exhibited the slowest decline of the concentration of the active moiety with levels of approximately $7 \mathrm{ng} / \mathrm{mL}$ at week 8 .

Abbreviations: ER, extended-release; LAl, long-acting injection.

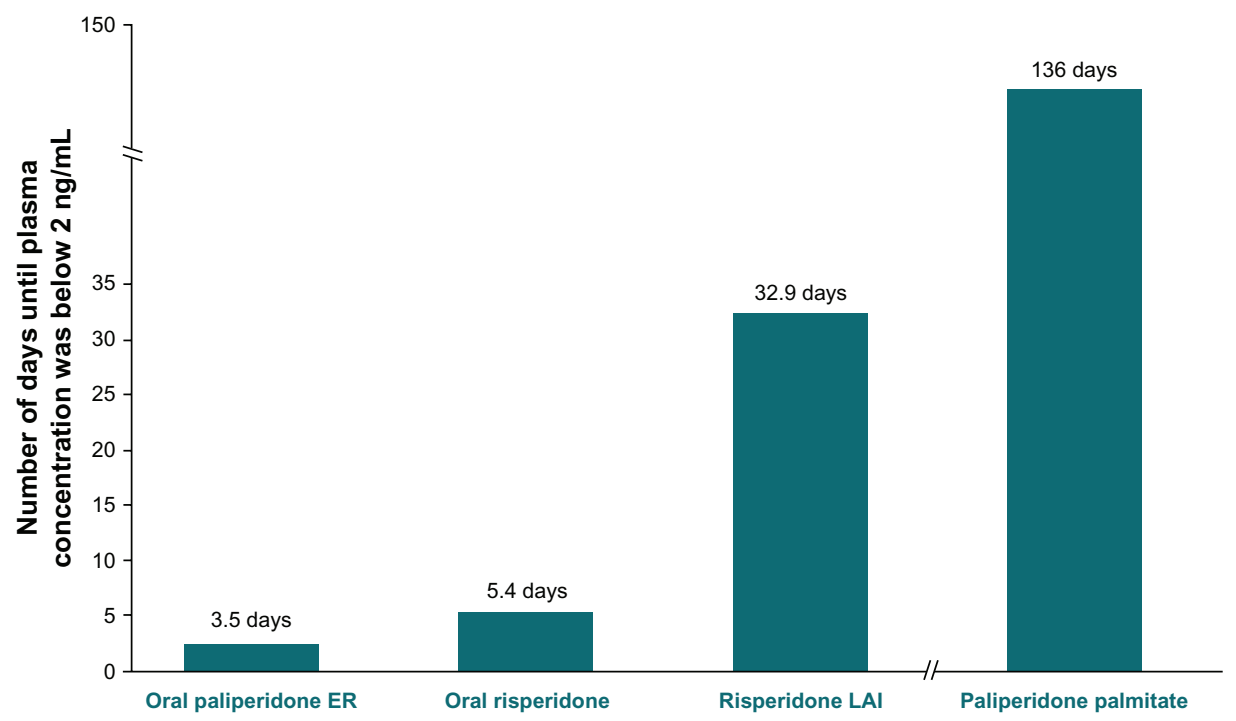

Figure 2 Number of days until active moiety plasma concentrations approach $2 \mathrm{ng} / \mathrm{mL}$, by agent.

Notes: Following treatment discontinuation, paliperidone palmitate long-acting injectable suspension required more than 4 months (I 36 days) for the plasma concentrations of the active moiety to approach $2 \mathrm{ng} / \mathrm{mL}$, with attainment of this threshold met less than one week after discontinuation of oral paliperidone ER (3.5 days) and oral risperidone (5.4 days), respectively, and approximately one month after discontinuation of risperidone LAl (32.9 days).

Abbreviations: ER, extended-release; LAl, long-acting injection. 
to a concentration of $7.2 \mathrm{ng} / \mathrm{mL}$ at week 8 , with this level not falling to below $2 \mathrm{ng} / \mathrm{mL}$ until day 136 (week 19.4).

\section{Scenario 2: one week of treatment interruption}

Interruption of treatment with either oral risperidone or oral paliperidone extended-release for one week resulted in concentrations of the active moiety that approached zero (Figure 3), with concentrations of the active moiety decreasing to $2 \%-11 \%$ of the steady-state concentration (Figure 4 ). Reinitiation of oral risperidone or oral paliperidone required a few days for the reattainment of steady-state concentrations of the active moiety (Figure 3 ).

Administration of risperidone long-acting injection at week -2 followed by a one-week interruption in the next scheduled dose affected the pharmacokinetic profile after a time delay, with concentrations of the active moiety being lower between weeks 4 and 5 despite treatment reinitiation after the one-week interruption (Figure 3). One week of interruption in risperidone long-acting injection administration was associated with a concentration of the active moiety decrease to $35 \%$ of the $\mathrm{C}_{\text {min,steady state }}$ (Figure 4 ).

A one-week interruption in paliperidone palmitate treatment was associated with a slight decrease in concentration of the active moiety (Figure 3 ), with the $\mathrm{C}_{\text {min,discontinuation }}$ reaching $90 \%$ of the $\mathrm{C}_{\text {min,steady state }}$ (Figure 4 ).

\section{Scenario 3: four weeks of treatment interruption}

As seen with one week of treatment interruption, 4 weeks of interruption of either oral antipsychotic agent was associated with a near zero concentration of the active moiety occurring

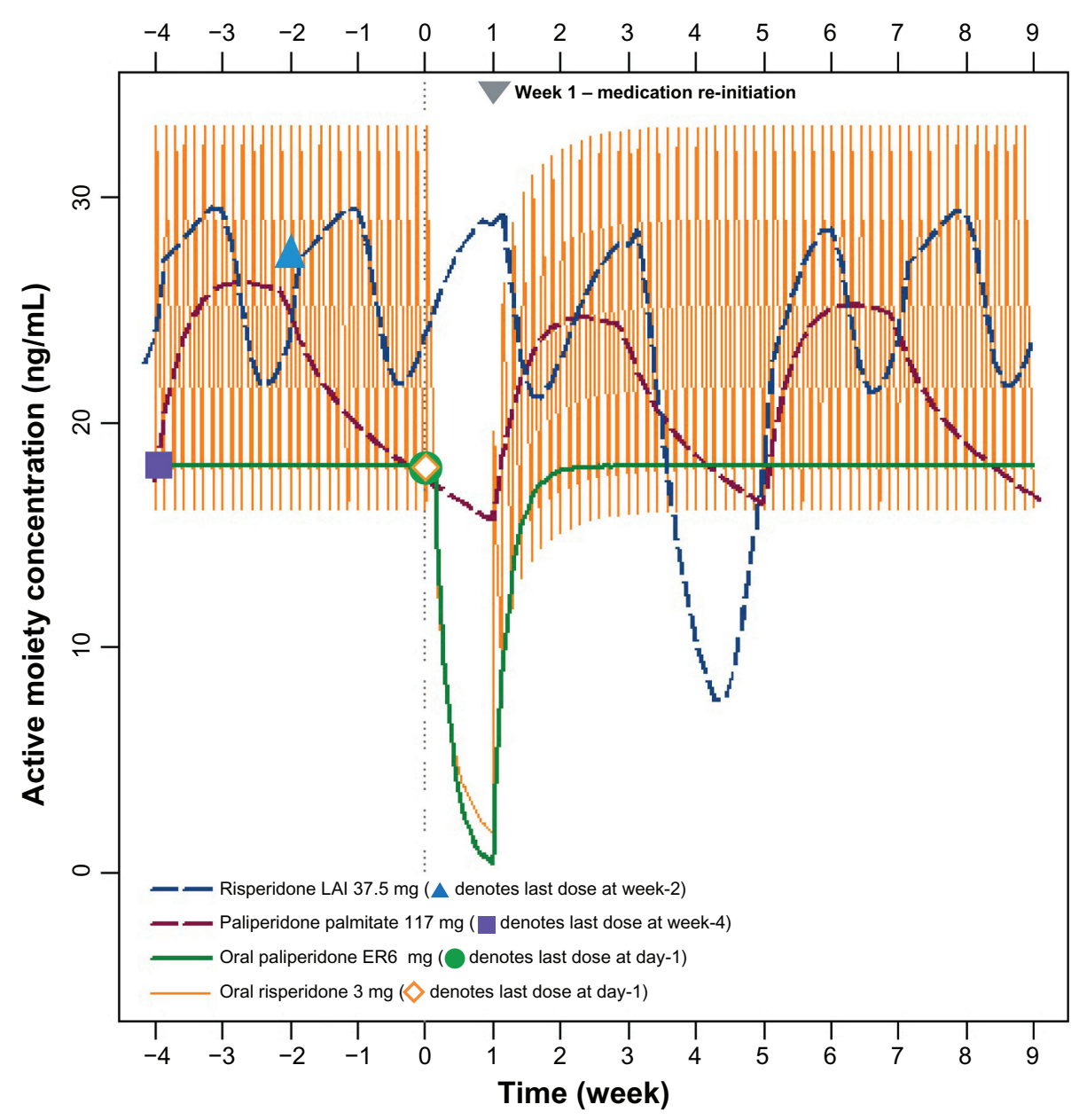

Figure 3 Active moiety concentrations following one week of treatment discontinuation (week 0 denotes administration time of next scheduled dose, which was missed). Notes: One week of interruption of either oral risperidone or oral paliperidone ER resulted in concentrations of the active moiety that approached zero. Reinitiation of oral risperidone or oral paliperidone ER required a few days for reattainment of steady-state concentrations of the active moiety. Administration of risperidone LAl at week -2 followed by a one-week interruption in the next scheduled dose resulted in active moiety concentrations being lower between weeks 4 and 5 despite treatment reinitiation at week I. A one-week interruption in paliperidone palmitate administration was associated with a slight decrease in active-moiety concentration, with one reinitiation dose producing a re-establishment of a near steady-state concentration. Abbreviations: ER, extended-release; LAl, long-acting injection. 


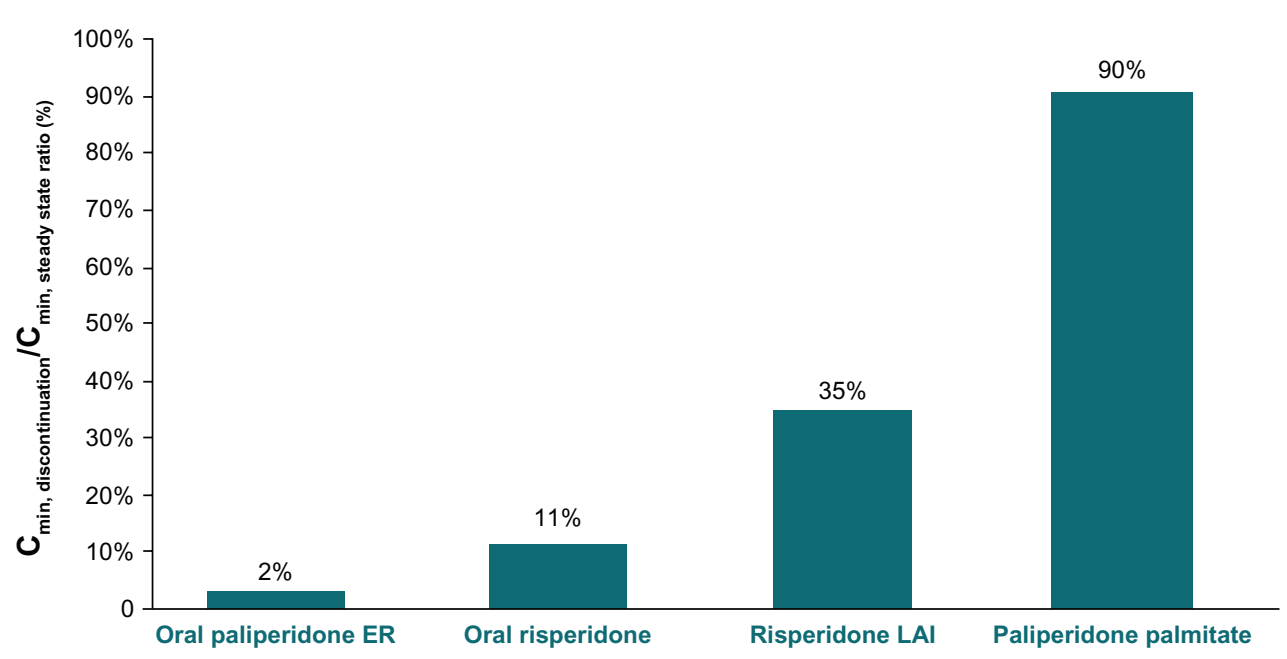

Figure 4 Lowest plasma concentration after one week of treatment discontinuation versus steady-state minimum concentration

Notes: One week of interruption of either oral risperidone or oral paliperidone ER resulted in the concentrations of the active moiety decreasing to $2 \%-1$ I $\%$ of the steadystate concentration. One week of interruption in risperidone LAI administration was associated with an active moiety concentration decrease to $35 \%$ of the steady-state minimum concentration. A one-week interruption in paliperidone palmitate treatment resulted in the plasma concentration being maintained at approximately $90 \%$ of the steady-state minimum concentration.

Abbreviations: ER, extended-release; LAI, long-acting injection.

within a few days of treatment interruption (Figures 5 and 6).

Following reinitiation, steady-state concentrations of the active moiety were re-established in approximately one week (Figure 5).

Four weeks of interruption in risperidone long-acting injection administration resulted in the active moiety plasma concentration decreasing to approximately $7.2 \mathrm{ng} / \mathrm{mL}$ at the end of week 4 (Figure 5). The active moiety plasma concentration in the risperidone long-acting injection group decreased to approximately $33 \%$ of the $\mathrm{C}_{\text {min,steady state }}$ concentration (Figure 6). Reinitiation of risperidone long-acting injection $37.5 \mathrm{mg}$ at weeks 4 and 6 , with oral risperidone $3 \mathrm{mg}$ once daily supplementation during weeks 4-7, resulted in a re-establishment of concentrations of the active moiety within a few days. However, as depicted in Figure 5, the concentration of the active moiety exhibits another decline between weeks 7 and 8 because the delayed release of risperidone long-acting injection does not begin until week 7 , while the risperidone from the oral supplementation rapidly washes out of the systemic circulation (ie, washout of risperidone from oral supplementation is much faster than the rise in concentrations from the reinitiation doses of risperidone long-acting injection).

Four weeks after the last administered dose of paliperidone palmitate, the concentrations of the active moiety continued a slow, steady decline (Figure 5), with the minimum concentration during this discontinuation period decreasing to $64 \%$ of the steady-state minimum concentration (Figure 6). Reinitiation of paliperidone palmitate $(117 \mathrm{mg}$ [75 $\mathrm{mg}$ eq] on days 28 and 35), without oral supplementation, produced a rapid reattainment of active moiety plasma concentrations (Figure 5).

\section{Discussion}

The three scenarios depicted in these pharmacokinetic model-based simulations approximate those that may be seen in clinical practice (ie, patients forgetting to take their oral dose[s] of antipsychotic medication or failing to return for their next injection of long-acting injection antipsychotic). The model-based simulations visually depict what would be anticipated to occur following complete treatment discontinuation or periods of treatment interruption. The long half-lives of the long-acting injectable agents may offer a longer time to subtherapeutic active moiety plasma concentrations and pharmacodynamically related symptom recurrence in those who fail to return for their next scheduled injection. As anticipated, paliperidone palmitate, a long-acting injectable antipsychotic with one of the longest half-lives, demonstrated the slowest decline in plasma concentrations. ${ }^{28,34,35}$

Antipsychotic pharmacokinetic profiles are generally reported from carefully controlled single-dose and multipledose studies. Clinical situations of nonadherence are difficult to study in clinical trials for ethical reasons, but the resulting plasma levels can be simulated using model-based tools. This analysis provides mathematically predicted profiles of antipsychotic plasma levels in clinical situations with the objective of aiding caregivers and patients by helping them make informed decisions regarding treatment nonadherence. 


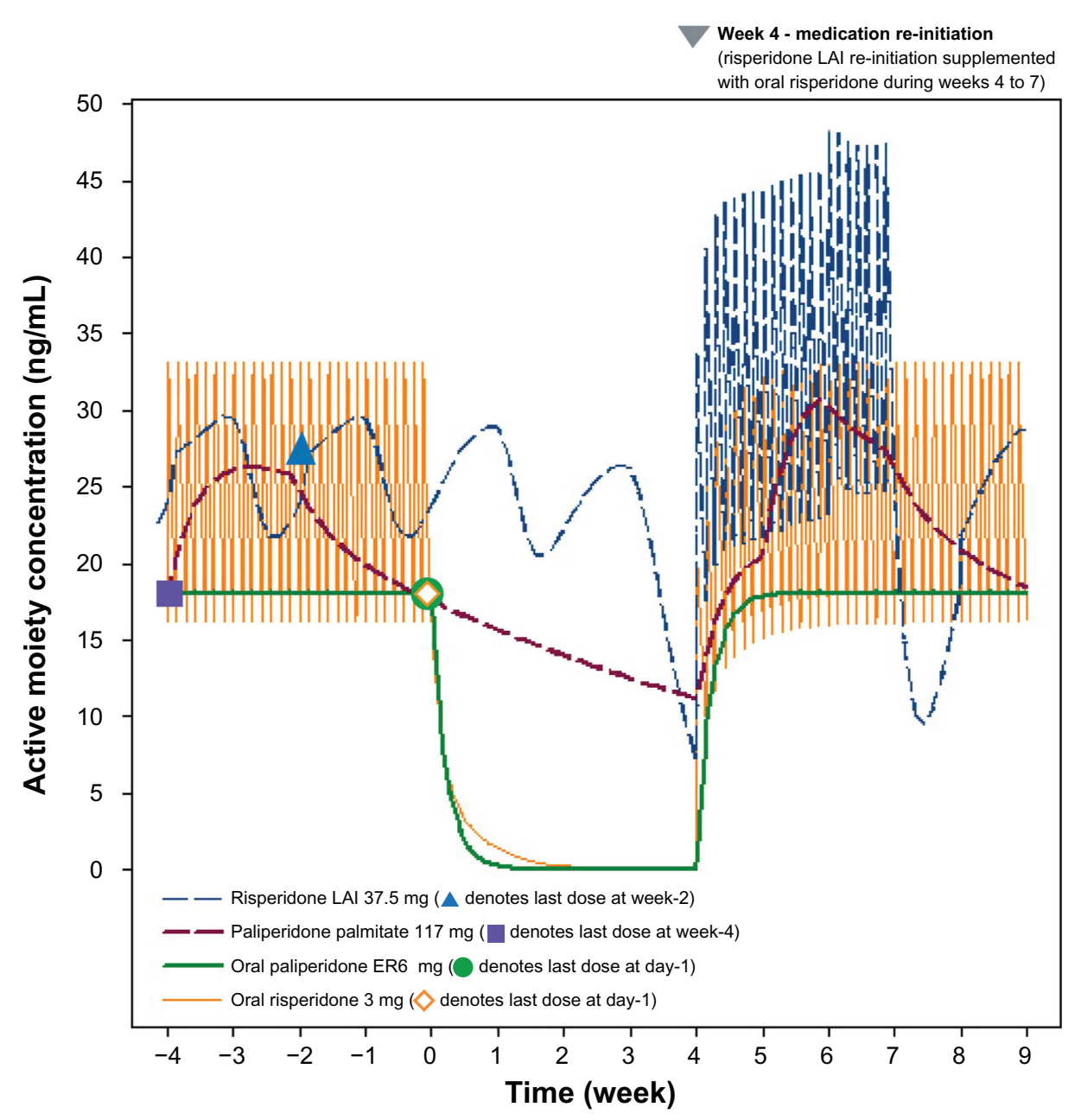

Figure 5 Active moiety concentrations following a 4-week treatment interruption and reinitiation (week 0 denotes administration time of next scheduled dose, which was missed).

Notes: Four weeks of interruption of either oral antipsychotic agent, risperidone or paliperidone, was associated with a near zero active moiety concentration, with these near zero levels occurring within a few days of treatment interruption. Following reinitiation, steady-state concentrations of the active moiety were re-established in approximately one week. Four weeks of interruption in risperidone LAl administration (ie, 6 weeks following the last administered dose) resulted in the plasma concentration of the active moiety decreasing to approximately $7.2 \mathrm{ng} / \mathrm{mL}$ at week 4 . Reinitiation of risperidone LAI 37.5 mg at weeks 4 and 6 , with oral risperidone 3 mg once-daily supplementation during weeks 4-7 resulted in a re-establishment of concentrations of the active moiety within a few days. However, due to the delayed-release characteristics of risperidone LAI, the active moiety concentration exhibits another decline between weeks 7 and 8 . Four weeks after the last administered dose of paliperidone palmitate, the concentrations of the active moiety continued a slow, steady decline. Reinitiation of paliperidone palmitate (II7 mg [75 mg eq] on days 28 and 35 ), without oral supplementation, produced a rapid reattainment of active moiety plasma concentrations.

Abbreviations: ER, extended-release; LAI, long-acting injection.

This is particularly important for the new long-acting injections because their mode of delivery and pharmacokinetics are designed to be different from the oral drugs and older intramuscular depot formulations. The three main scenarios assessed were complete discontinuation, short-term interruption, and longer-term interruption. Each scenario provides useful insight about consequences of nonadherence, ie, the treatment discontinuation scenarios help with the assessment of the magnitude of time that patients can go without a dose, until their concentrations reach a critical threshold at which response could be compromised (ie, the drug's "forgiveness"). The discontinuation assessments indicate that following treatment discontinuation, paliperidone palmitate long-acting injection requires more than 4 months to approach a critical threshold of $2 \mathrm{ng} / \mathrm{mL}$, while this threshold was reached in less than one week after discontinuation of oral paliperidone extended-release and oral risperidone, and approximately one month after discontinuation of risperidone long-acting injection (Figure 2). The short-term and longer-term interruption scenarios suggest that managing the possible clinical effects of nonadherence with risperidone long-acting injection may not be easy, because the effect of even a single delayed dose may not be evident for $4-5$ weeks due to its complex absorption kinetics. Because these delayed changes in drug levels may affect drug efficacy, caregivers need to be aware of the 3-week lag time between injection 


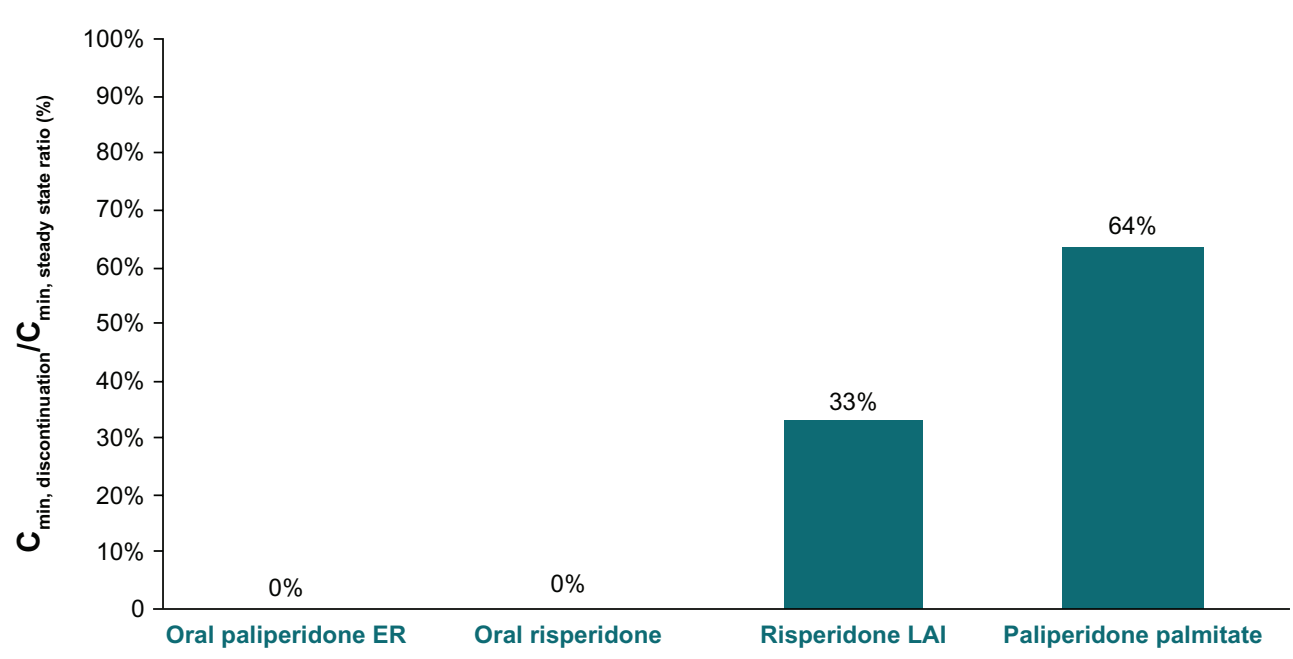

Figure 6 Lowest plasma concentration after 4 weeks of treatment discontinuation versus steady-state minimum concentration (\%).

Notes: Four weeks of interruption of either oral antipsychotic agent, risperidone or paliperidone, was associated with zero concentration of the active moiety. The plasma concentration of the active moiety in the risperidone LAI group decreased to approximately $33 \%$ of the steady-state minimum concentration following 4 weeks of treatment discontinuation. Four weeks after the last administered dose of paliperidone palmitate the minimum active moiety concentration decreased to $64 \%$ of the steady-state minimum concentration.

Abbreviations: ER, extended-release; LAI, long-acting injection.

and drug release for risperidone long-acting injection. This lag time makes it difficult to anticipate the pharmacokinetic profile for risperidone long-acting injection during situations of nonadherence and the graphical representations provided in the current analysis can serve as a useful visual aid. Finally, longer-term interruptions require reinitiation methods that are specific to each drug, and paliperidone palmitate longacting injection allows a simple two-dose reinitiation strategy without the need for oral supplementation.

The reinitiation of antipsychotic treatment in the scenarios that simulated a one-week or 4-week lapse in treatment adherence, especially in cases of long-acting injection treatment, poses challenges. Reinitiation of oral agents is straightforward because it resembles de novo treatment but attainment of therapeutic levels takes up to one week. In the case of long-acting injectable agents, it is desirable to attain therapeutic plasma levels quickly; however, reinitiation scenarios, such as with risperidone long-acting injection, which require oral supplementation for the first 3 weeks, are to some extent more complex. Discontinuation or interruption of risperidone long-acting injection is associated with a delay in the time for plasma concentrations to decrease, with the effect of these scenarios being depicted graphically. These delays may represent a time window for clinicians to intervene in the event of nonadherence to manage the delayed decreases in plasma concentration with oral supplementation. It is worth noting that oral supplementation during reinitiation has not been tested in clinical trials. The sustained concentrations of paliperidone in the 4-week interruption simulation using paliperidone palmitate resulted in $\mathrm{C}_{\text {min,discontinuation }}$ of $64 \%$ of $\mathrm{C}_{\text {min,steady state }} 4$ weeks after the missed injection. The clinical effect of these concentrations is uncertain. However, as noted by some, the long time to symptom recurrence among patients administered paliperidone palmitate who were then switched to placebo in relapse prevention studies suggests that the long half-life for paliperidone palmitate may allow more time to follow-up if a dose is delayed. ${ }^{19}$ Furthermore, steady-state paliperidone concentrations can be rapidly reestablished in this scenario using the recommended reinitiation strategy (administration of two paliperidone palmitate injections, each at the last administered dose and given one week apart, at weeks 4 and 5). ${ }^{29}$

Limitations of the simulations for these potential clinical scenarios are that they are model-based and that changes in active moiety plasma concentrations do not necessarily correlate strongly with pharmacodynamic changes in the efficacy or safety of an antipsychotic. However, these pharmacokinetic model-based simulations suggest that longacting injectable formulations of antipsychotic agents offer at least a pharmacokinetic benefit in patients who are suspected of or have demonstrated incomplete medication adherence behavior. The more sustained plasma concentrations of longacting injections may provide a wider window of opportunity to re-establish treatment without symptom recurrence or relapse compared with oral antipsychotics. Among longacting injections, paliperidone palmitate has one of the longest apparent half-lives, and therefore may provide a longer dosing window for follow-up if a dose is delayed. 
Reinitiation of paliperidone palmitate is relatively easy, with steady-state plasma concentrations rapidly reattained following administration of one dose in cases of one week of treatment interruption or two doses given one week apart in cases of 4 weeks of treatment interruption.

\section{Disclosures}

MNS is an employee of Janssen Research and Development, Raritan, NJ. D-JF, JKS, and LA are employees of Janssen Scientific Affairs, Titusville, NJ, and BR is an employee of Janssen Research and Development, Beerse, Belgium. At the time of this analysis, JS was an employee of Janssen Scientific Affairs, Titusville, NJ. The authors acknowledge the editorial support services provided by Susan Ruffalo of MedWrite Inc, Newport Coast, CA. This research was presented at the 14th Annual Meeting of the College of Psychiatric and Neurologic Pharmacists, May 1-4, 2011, Phoenix, AZ, and at the 51st Annual New Clinical Drug Evaluation Unit New Research Approaches for Mental Health Interventions Meeting, June 13-16, 2011, Boca Raton, FL. This pharmacokinetic model-based simulation study was supported by Janssen Scientific Affairs, Titusville, NJ, and is not registered at ClinicalTrials.gov.

\section{References}

1. Lehman AF, Lieberman JA, Dixon LB, et al. Practice guideline for the treatment of patients with schizophrenia, second edition. Am J Psych. 2004;161(2 Suppl):1-56.

2. Kane JM. Treatment adherence and long-term outcomes. CNS Spectrums. 2007;12(10 Suppl 17):21-26.

3. Marshall M, Rathbone J. Early intervention for psychosis. Cochrane Database Syst Rev. 2006;6:CD004718.

4. Bhanji H, Chouinard G, Margolese HC. A review of compliance, depot intramuscular antipsychotics and the new long-acting injectable atypical antipsychotic risperidone in schizophrenia. Eur Neuropsychopharmacol. 2004; 14:87-92.

5. Masand PS, Roca M, Turner MS, Kane JM. Partial adherence to antipsychotic medication impacts the course of illness in patients with schizophrenia: a review. Prim Care Companion J Clin Psychiatry. 2009;11:147-154.

6. Weiden PJ, Zygmunt A. The road back: working with the severely mentally ill. Medication noncompliance in schizophrenia. J Pract Psychiatr Behav Health. 1997;3:106-110.

7. Tiihonen J, Haukka J, Taylor M, Haddad PM, Patel MX, Korhonen P. A nationwide cohort study of oral and depot antipsychotics after first hospitalization for schizophrenia. Am J Psychiatry. 2011;168:603-609.

8. Morken G, Widen JH, Grawe RW. Non-adherence to antipsychotic medication, relapse and rehospitalisation in recent-onset schizophrenia. BMC Psychiatry. 2008;8:32-39.

9. Schooler NR. Relapse and rehospitalization: comparing oral and depot antipsychotics. J Clin Psychiatry. 2003;64 Suppl 16:14-17.

10. Kozma CM, Slaton T, Dirani R, Gopal S, Fastenau J, Hough D. Changes in schizophrenia-related health care resource utilization among patients receiving paliperidone palmitate: results of a 52-week clinical trial. Curr Med Res Opin. 2011;27:1603-1611.
11. Subotnik KL, Nuechterlein KH, Ventura J, et al. Risperidone nonadherence and return of positive symptoms in the early course of schizophrenia. Am J Psychiatry. 2011;168:286-292.

12. Weiden PJ, Kozma C, Grogg A, Locklear J. Partial compliance and risk of rehospitalization among California Medicaid patients with schizophrenia. Psychiatr Serv. 2004;55:886-891.

13. Keith SJ, Kane JM. Partial compliance and patient consequences in schizophrenia: our patients can do better. $J$ Clin Psychiatry. 2003;64:1308-1315.

14. Kane JM, Garcia-Ribera C. Clinical guideline recommendations for antipsychotic long-acting injections. $\mathrm{Br} J$ Psychiatry. 2009;195:63-67.

15. Robinson DG. Medication adherence and relapse in recent-onset psychosis. Am J Psychiatry. 2011;168:240-242.

16. Zhu B, Ascher-Svanum H, Shi L, Faries D, Montgomery W, Marder SR. Time to discontinuation of depot and oral first-generation antipsychotics in the usual care of schizophrenia. Psychiatr Serv. 2008;59:315-317.

17. Kramer M, Simpson G, Maciulis V, et al. Paliperidone extended-release tablets for prevention of symptom recurrence in patients with schizophrenia: a randomized, double-blind, placebo-controlled study. J Clin Psychopharmacol. 2007;27:6-14.

18. Hough D, Gopal S, Vijapurkar U, et al. Paliperidone palmitate maintenance treatment in delaying the time-to-relapse in patients with schizophrenia: a randomized, double-blind, placebo-controlled study. Schizophr Res. 2010;116:107-117.

19. Markowitz M, Turkoz I, Fu DJ, et al. Post hoc comparative effectiveness study of paliperidone palmitate vs paliperidone ER: an indirect comparison from placebo-controlled relapse prevention studies. Presented at the American College of Neuropsychopharmacology 49th Annual Meeting, December 5-9, 2010, Miami Beach, FL.

20. Wilson WH. A visual guide to expected blood levels of longacting injectable risperidone in clinical practice. $J$ Psychiatr Pract. 2004;10:393-401.

21. Risperdal ${ }^{\circledR}$ (risperidone, full prescribing information). Titusville, FL: Janssen, Division of Ortho-McNeil-Janssen Pharmaceuticals Inc; 2011.

22. Invega ${ }^{\circledR}$ (paliperidone, full prescribing information) Titusville, FL: Janssen, Division of Ortho-McNeil-Janssen Pharmaceuticals Inc; 2011.

23. Boom S, Talluri K, Janssens L, et al. Single- and multiple-dose pharmacokinetics and dose proportionality of the psychotropic agent paliperidone extended release. J Clin Pharmacol. 2009;49: $1318-1330$.

24. Cirincione B, Redman M, Fiedler-Kelley J, Ludwig E, Vermeulen A. Population pharmacokinetics of paliperidone ER in healthy subjects and patients with schizophrenia. Clin Pharmacol Ther. 2007;81:S19.

25. Thyssen A, Vermeulen A, Fuseau E, Fabre MA, Mannaert E. Population pharmacokinetics of oral risperidone in children, adolescents and adults with psychiatric disorders. Clin Pharmacokinet. 2010;49: 465-478.

26. Risperda ${ }^{\circledR}$ Consta $^{\circledR}$ (risperidone, full prescribing information.) Titusville, FL: Janssen, Division of Ortho-McNeil-Janssen Pharmaceuticals Inc; 2011.

27. Gefvert O, Eriksson B, Persson P, et al. Pharmacokinetics and D2 receptor occupancy of long-acting injectable risperidone (Risperda ${ }^{\circledR}$ Consta $^{\circledR}$ ) in patients with schizophrenia. Int J Neuropsychopharmacol. 2005;8:27-36.

28. Samtani A, Vermeulen A, Stuyckens K. Population pharmacokinetics of intramuscular paliperidone palmitate in patients with schizophrenia: a novel once-monthly long-acting formulation of atypical antipsychotic. Clin Pharmacokinet. 2009;48:585-600.

29. Invega ${ }^{\circledR}$ Sustenna ${ }^{\circledR}$ (paliperidone palmitate) extended-release injectable suspension prescribing information. Titusville, NJ: Janssen, Division of Ortho-McNeil-Janssen Pharmaceuticals Inc; 2011. 
30. Cleton A, Rossenu S, Hough D, et al. Assessment of the dose proportionality of paliperidone palmitate $25,50,100$, and $150 \mathrm{mg}$ eq. A new long-acting injectable antipsychotic following administration in the deltoid or gluteal muscles. Clin Pharmacol Ther. 2008;83:S31.

31. Bishara D. Once-monthly paliperidone injection for the treatment of schizophrenia. Neuropsychiatr Dis Treat. 2010;6:561-572.

32. Eerdekens M, Karcher K, Remmerie B, et al. Pharmacokinetics and tolerability of long-acting injectable risperidone in schizophrenia. Schizophr Res. 2004;70:91-100.

33. Product monograph. Invega ${ }^{\circledR}$ Sustenna ${ }^{\circledR}$ paliperidone palmitate prolonged-release injectable suspension. Toronto, Ontario: Janssen-Ortho Inc; 2010.
34. Samtani MN, Gopal S, Gassmann-Mayer C, Alphs L, Palumbo JM Dosing and switching strategies for paliperidone palmitate: based on population pharmacokinetic modeling and clinical trial data. CNS Drugs. 2011;25:829-845.

35. Sheehan JJ, Reilly KR, Fu DJ, Alphs L. Comparison of the peak-totrough fluctuation in plasma concentration of long-acting injectable antipsychotics and their oral equivalents. Poster 126 presented at $23 \mathrm{rd}$ Annual US Psychiatric and Mental Health Congress Conference and Exhibition, November 18-21, 2010, Orlando, FL. 


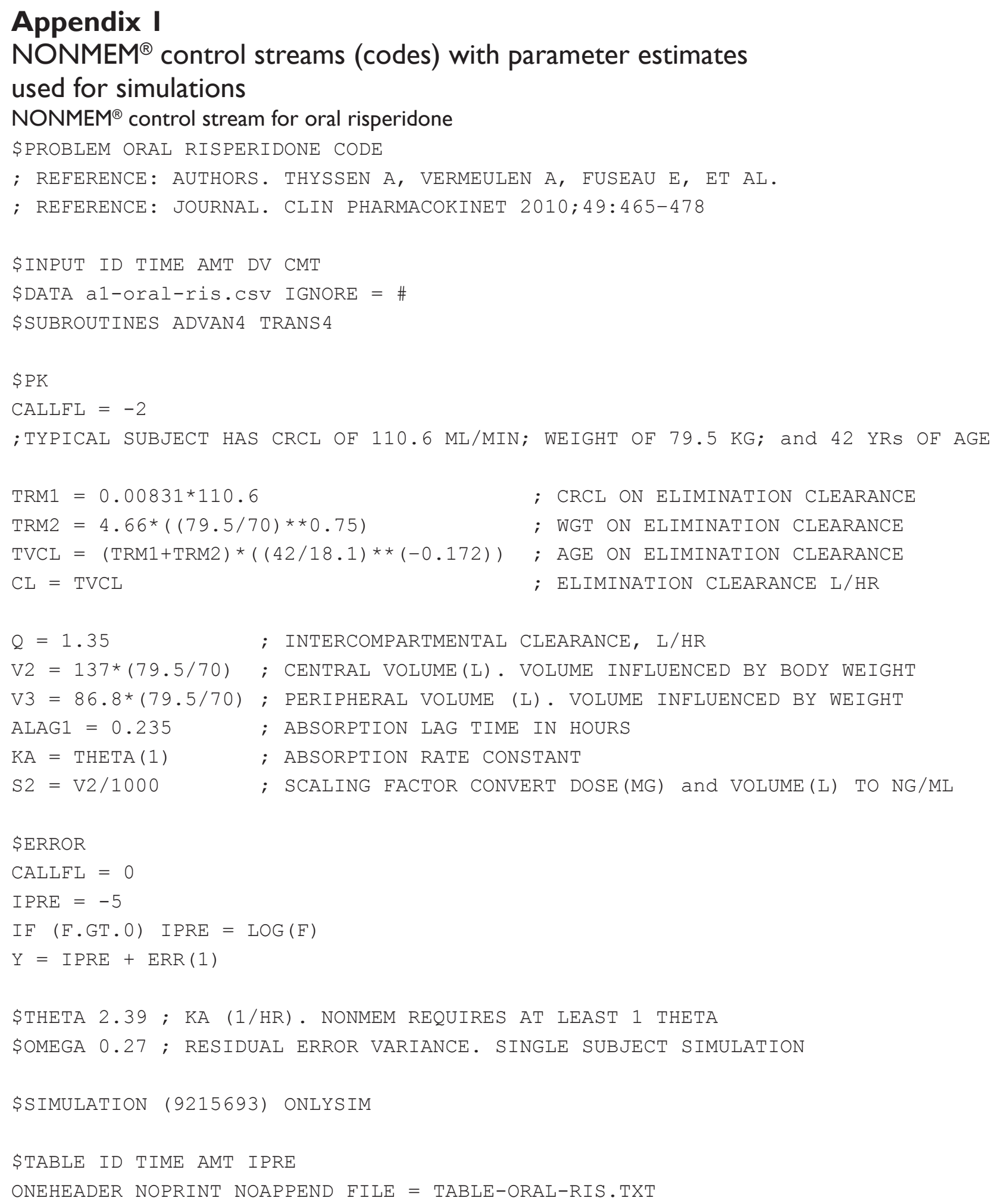




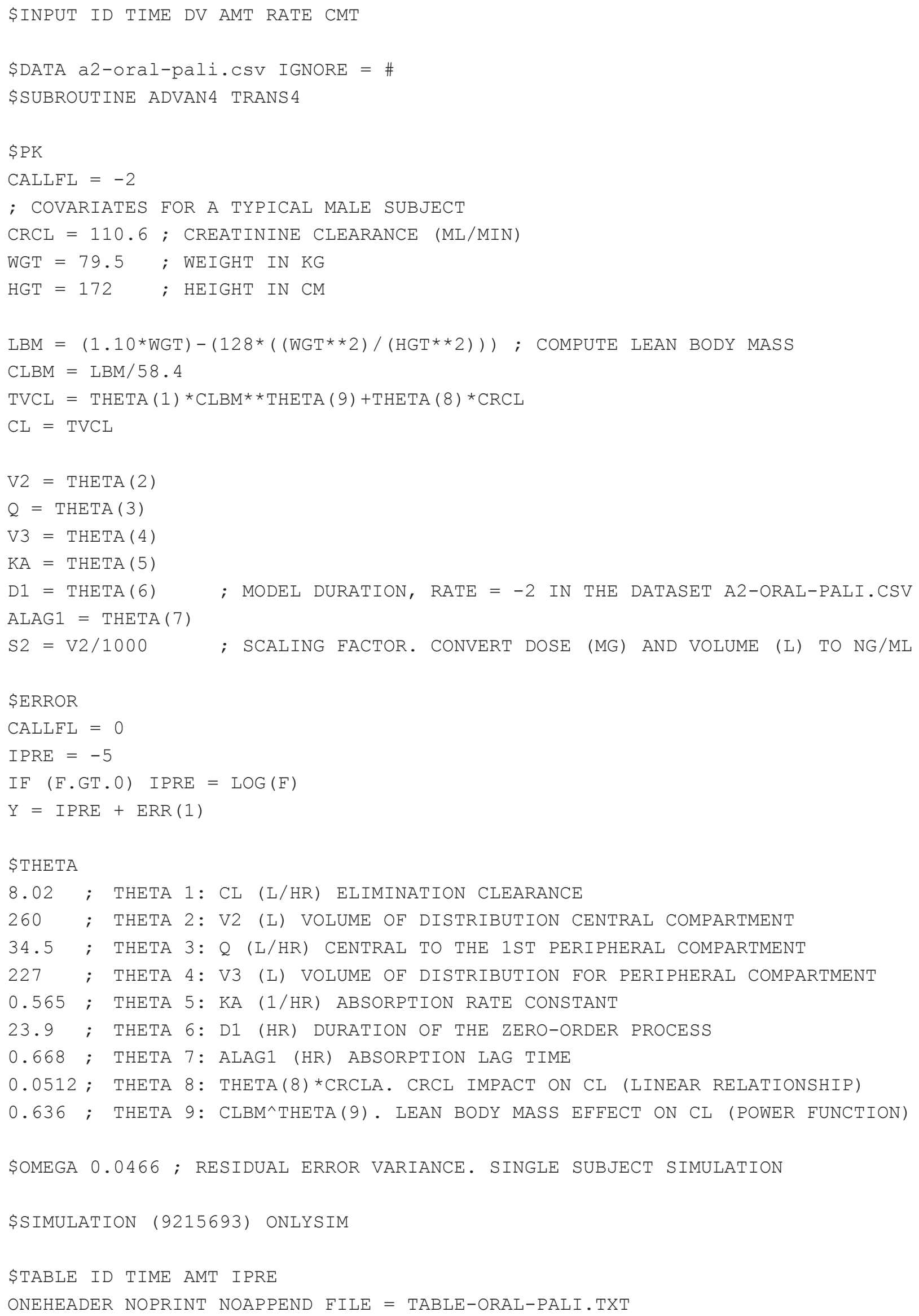




\section{NONMEM ${ }^{\circledR}$ control stream for risperidone LAI}

\$PROBLEM RISPERIDONE LAI

; REFERENCE: AUTHORS. SAMTANI MN, GOPAL S, SLIWA JK, ET AL.

; REFERENCE: POSTER PRESENTEd AT PAGE; BERLIN, GERMANy, JUNE 8-10, 2010.

; REFERENCE: PAGE 19 (2010) Abstr 1839 [http://www.page-meeting.org/?abstract=1839]

Samtani et al. CNS Drugs. 2011;25(10):829-45.

\$INPUT ID TIME AMT CMT RATE DV

\$DATA a4-ris-consta.CSV IGNORE = \#

\$SUB ADVAN5

\$MODEL

$\mathrm{COMP}=(\mathrm{DOSE} 1)$

$\mathrm{COMP}=(\mathrm{DOSE} 2)$

$\mathrm{COMP}=(\mathrm{DOSE} 3)$

COMP $=($ INTER 1$)$

COMP $=($ INTER2 $)$

COMP $=($ CENTRAL DEFOBS $)$

$\$ P K$

CALLFL $=-2$

$\mathrm{CL}=4.25$

$\mathrm{V} 6=244.66$

; ELIMINATION CLEARANCE (L/HR)

$\mathrm{KA} 1=10.775$

; CENTRAL VOLUME (L)

$\mathrm{KA} 2=0.005$

; ABSORPtion RATE CONSTANTS (1/HR) 1ST PROCESS

$\mathrm{KA} 3=0.023$

; ABSORPTION RATE CONSTANTS (1/HR) 2 ND PROCESS

$\mathrm{F} 1=0.0215$

; ABSORPTION RATE CONSTANTS (1/HR) 3RD PROCESS

$\mathrm{F} 3=0.78$

; FRACTION OF DOSE ABSORBED VIA 1ST PROCESS

$\mathrm{F} 2=\mathrm{THETA}(1)-\mathrm{F} 1-\mathrm{F} 3$

; FRACTION OF DOSE ABSORBED VIA 3RD PROCESS

ALAG2 $=8.465$

; FRACTION OF DOSE ABSORBED VIA 2 ND PROCESS

ALAG3 $=543.19$

; LAG TIME (HR) SECOND PROCESS

$\mathrm{D} 2=416.695$

; LAG TIME (HR) THIRD PROCESS

D3 $=271.535$

; DURATION (HR) OF ZERO ORDER INPUT, 2 ND PROCESS

$\mathrm{S} 6=\mathrm{V} 6 / 1000$

; DURATION (HR) OF ZERO ORDER INPUT, 3RD PROCESS

; CONVERT DOSE (MG) and VOLUME (L) TO NG/ML

$\mathrm{K} 16=\mathrm{KA} 1$

$\mathrm{K} 24=\mathrm{KA} 2$

$\mathrm{K} 46=\mathrm{KA} 2$

$\mathrm{K} 35=\mathrm{KA} 3$

$\mathrm{K} 56=\mathrm{KA} 3$

$\mathrm{K} 60=\mathrm{CL} / \mathrm{V} 6$

\$ERROR

CALLFL $=0$

$I P R E=-5$

IF (F.GT.0) IPRE = LOG (F)

$Y=\operatorname{IPRE}+E R R(1)$ 


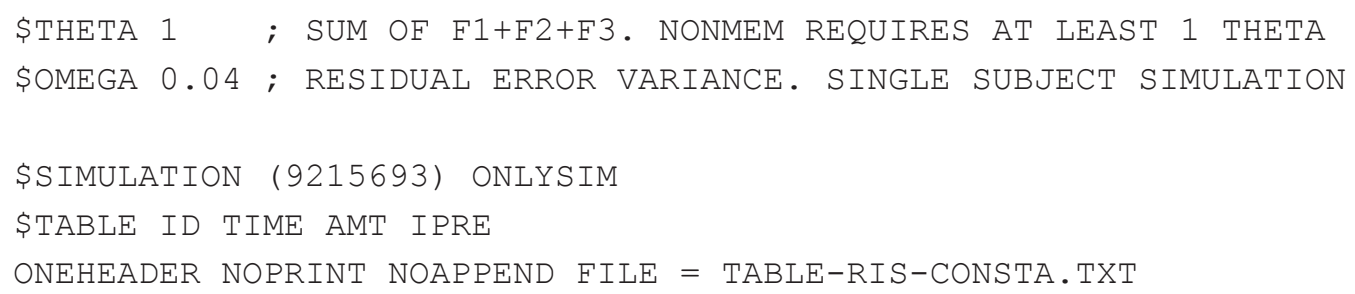

\section{$\mathrm{NONMEM}^{\circledR}$ control stream for paliperidone palmitate}

\$PROBLEM PALI PALMITATE SIMULATION

; REFERENCE: Samtani A, Vermeulen A, Stuyckens K. Clin PK 2009;48:585-600. \$INPUT ID TIME DV AMT CMT RATE 


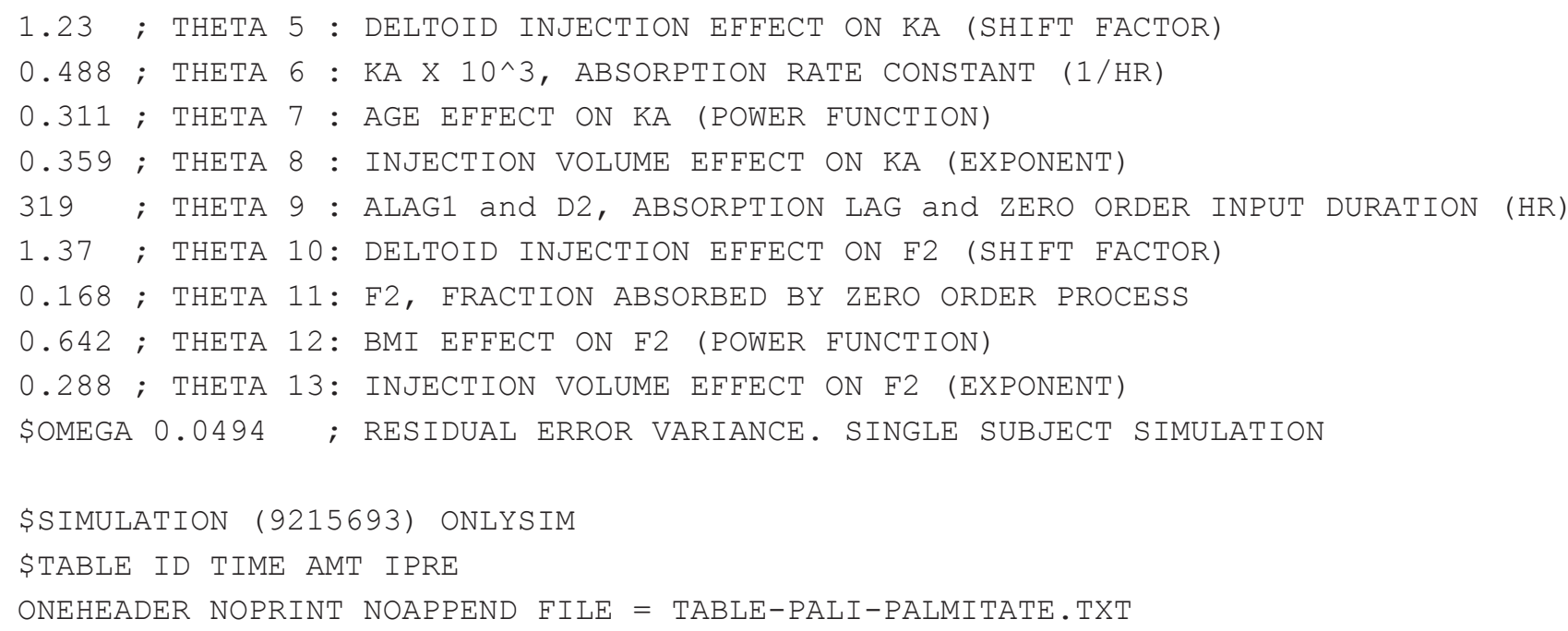

\section{Publish your work in this journal}

Clinical Pharmacology: Advances and Applications is an international, Visit http://www.dovepress.com/testimonials.php to read real quotes

peer-reviewed, open access journal publishing original research, reports, from published authors.

reviews and commentaries on all areas of drug experience in humans.

The manuscript management system is completely online and includes

a very quick and fair peer-review system, which is all easy to use.

Submit your manuscript here: http://www.dovepress.com/clinical-pharmacology-advances-and-applications-journal 